\title{
Multi-Agent-Based Simulation of Decentralized Energy Systems
}

\author{
Klaus-Peter Fähnrich, Stefan Kühne* and Axel Hummel ${ }^{*}$ \\ Universität Leipzig, Augustusplatz 10, 04109 Leipzig \\ *Corresponding author
}

\begin{abstract}
Future energy systems require decentralized regulatory approaches. Aggregates, equipped with appropriate sensors, can behave independently for the purposes of system stability. To explore and evaluate appropriate control mechanisms a multiagent-based simulation will be developed. It enables realistic simulations of electricity grids and decentralized consumption, storage and production units as well as provides the basis for further research whether the concept in scale could be successful. We describe the general architecture of such a simulation approach in comparison to existing literature.
\end{abstract}

Keywords-multi-agent-based simulation; decentral energy systems

\section{INTRODUCTION}

The integration of renewable energies such as photovoltaic and wind energy into energy supply systems places new requirements on actors, technology and system architecture. The volatile and decentralized energy production and feed in require new concepts for regulation and control. Currently these challenges are addressed by network optimization, network expansion, the development of new storage technologies and the use of new flexibility in energy consumption. Consumers are thereby integrated into centralized control mechanisms with the help of intelligent smart meters to enable an individual control. Following existing grid mechanism, these concepts are extended in particular in small consumption of industrial, commercial and household customers.

The centralized control approach based on smart grid and smart meter technologies is only manageable with high IT and communication efforts. The requirements are further increased by necessary redundancy of safety-critical applications, such as the provision of regulation energy. The approach is therefore expensive, raises data privacy and acceptance issues and requires additional energy to operate the required control systems.

A promising and complementary approach is the use of decentralized control mechanisms in energy supply systems. In electricity networks intelligent actors, equipped with appropriate sensors, could behave independently and appropriately in terms of system safety by measuring electricity parameters without having to get detailed objectives from central control facilities. Decentralized control approaches can be realized with a substantially lower amount of communication infrastructure and with no direct central control of each actor. In particular, it decreases the demands on bandwidth, timing and availability of the communication paths and the downstream control. Ideally, at the lowest power levels a communication link and a direct control interface can even be omitted.

In this paper we describe a multi-agent based simulation approach to explore decentralized control mechanisms in energy systems. It should be evaluated whether a system composed of decentralized actors can show an emergent behavior that leads to stable system states despite strong fluctuation influences by renewable energies and new consumer classes.

\section{A Multi-Agent-Based SimUlation ApPROACH}

To demonstrate the basic behavior and possible implementations of decentralized energy systems in terms of realistic simulation studies a multi-agent-based simulation approach will be developed. In our approach, the technical and economic system elements are represented by three different agent meta classes.

The first meta class "network components" covers technical units in the grid of the reference object. Agent classes of this meta class include local production plants, storage, consumer, and the electrical grid itself with its respective components. The aim of this model layer is to cover physical processes in the power grid (represented as changes in frequency, voltage, etc.) and to dynamically simulate the changes in the grid as a function of the behavior of its aggregates (harmonics and dynamic couplings).

The "hardware" agents are supplemented by a second agent meta class for communication systems. Agents ("communication agents") of this meta class interlink other agents from a communication perspective.

The third agent meta class ("player agent") covers economic actors such as private households, commercial customers, network operators, power plant operators, market places, etc. They define the requirements for the operation of the system elements, assess their economic success and, accordingly, change their behavior.

The separation of the three different levels allows the analysis of different control strategies within the same system. On the other hand, it provides the basis to reflect and evaluate stress situations, such as the failure of the communication systems within a black start-up scenario. 
The multi-agent system should be implemented as a holonic system [1]. This approach is based on the idea that a single agent (holon) can communicate with other agents to a group, a so-called (super)-holon. Even this (super)-holon can merge with other agents to a more complex (super)-holon. The recursive definition of such a model allows a natural illustration of the heterogeneous structures of energy systems. The special characteristic of the approach is that a (super)holon is perceived as a single agent to the outside and not as a group of agents. Responsible for this is the head of the (super)holon, which is either embodying the included agents or is represented by a new agent to be produced.

In this way a single household could represent an agent system consisting of autonomous agents (consumers, producers) and appeal as a single agent at a higher level (e.g. distribution grid). The distribution grid itself could then in turn be an individual agent in an agent system of distribution or regional grids.

Aim of the multi-agent-based simulation approach is to examine the following scenarios:

1) Tests of system stability in regular operation mode, no stability-stressing, emergent behavior of agents such as sudden, chaotic swinging;

2) System behaviour within overload, lack of energy or technical faults (e.g. short-circuit);

3) Stability in stress situations induced by consumers or by feedin of renewable energy plants;

4) Stability in scaling the number of autonomously acting units in the network.

\section{REQUIREMENTS}

To achieve these objectives the following basic requirements are relevant:

\section{A. Stability of the Overall System}

An exact physical simulation of power grids is the basis for a realistic modeling and for answering the question of stability of the overall system. Therefore the physical simulation plays a key role in our approach. It is necessary to ensure that the dynamic behavior of electrical or electro-mechanical systems can be mapped with sufficient precision and that the basic law of energy conservation is fulfilled in dynamic processes complies with peak loads and load changes. Furthermore, energy losses in the power system caused for example by heat have to be mapped correctly.

Since the overall system behavior results from the interaction of the agents of all three meta-levels, it is necessary to consider also the entire system from the physical layer up to the electricity price information in electricity markets. For example, in practice, the ramping processes arising from energy trading every full hour (trade rhythm) lead to extensive fluctuations in the grid frequency.

\section{B. Accuracy of the Physical Simulation}

The technical and physical operation of the power system includes diverse stressful situations e.g. frequency deviations, overload, transients, harmonics, etc. which result in different selective and adapted reactions e.g. overcurrent trip, frequency-based load shedding, etc. in order to prevent damages. Moreover, large requirement changes to the power grid may result in new “emergent” effects.

The "guidelines" in the energy system must therefore be constantly reviewed and, if necessary, revised or newly developed. It's not just about the device behavior, physical (limit) values etc. but in particular also to the chronological sequence of events (e.g. crises, load changes, etc.). The agent simulation system must therefore be able to imitate such operations sufficiently precisely.

Conventional physical simulations use special solver for such cases, which dynamically adjust the time steps in the calculation of the simulation in order to capture high frequency operations accurately. Discrete events, such as switching operations can therefore be taken into account with their exact time of entry. So continuous physical systems with discrete events can be simulated very accurately.

For agent-based simulation systems no such solver mechanisms exist. Without a solver the calculation of the physical solution is done either in constant time steps or driven by the requests of the control (ICT model level). Questions about network operation in the event of a crisis (e.g. voltage drops versus short-circuit power deployment) could possibly get lost through the large time steps of the simulation clock and therefore not taken into account.

\section{Development of a Comprehensive System Architecture}

Another goal of the multi-agent-based approach is to enhance the system architecture of the physical level out to the actor level and thereby be able to represent real communication conditions (communication level). In achieving this, we intent to specify and implement prototypical actor classes such as private households, businesses and industries but also system-specific players such as energy suppliers, production plant operators and network operators within the meta class of actors. The same must be done for the communication level, whereby the communication paths between the actors and the physical level have to be mapped. The various communication paths should be structured into several agent classes that represent different communication approaches with specific situational availabilities, reliabilities, bandwidths, time performance and energy consumption. Examples of such different communication approaches are physical switches, ripple control or classical cable or radiobased ICT approaches.

This activity aims at providing the necessary basis for the development of methods, algorithms and concepts in order to investigate the following questions:

- What information can be processes decentralized and locally and implemented in which actions and how? 
- What information may need to be communicated in the system (e.g. not physical expectations)?

- Black start-up ability and isolated operation

- $\quad$ System services

- Automatic load balancing and efficiency optimization of the network

- Impact of the grid integration of a large number of renewable distributed generation and distributed storage

- Effects of different energy-related regulatory decisions

- Models for collaboration between different market participants on common data platforms

Data aggregation and analysis:

- For network monitoring and network expansion planning

- For the analysis of economic relevant data, for example, the profitability of different production strategies to issues of urban planning

- To integrate existing and future distribution models e.g. aggregators for energy storage / virtual power plants

- For socio-economic interaction with the network (conventional Smart Grid, Smart Metering, Smart Home, Smart Markets, energy services)

- Extensibility of the agent-based network control on heating and gas networks or transport systems

\section{RELATED WORK}

Multi-agent systems have already been successfully used for the analysis of energy markets in various contexts. [1] provides a systematic overview of the state of the art regarding the use of multi-agent systems in the field of simulation of electricity markets. The authors come to the conclusion that the use of multi-agent systems is appropriate, in order to gain insights into the design and development of electricity markets. The most important challenge is the access to real data, for modeling of concrete power plants as well as for modeling the demand side. In most models this results in the "garbage in, garbage out" phenomenon - the challenge of validating the results. Another problem is the validation of behavior and learning effects of individual agents in the simulation.

A thorough investigation of different agent-based models for energy markets was collected and classified in [2]. The authors have categorized 31 different publications. However, only four considered that the network transmission could be a limiting factor and could have relevant influence on the analysis. However, even these four publications considered the power only as a DC power. Since real networks are almost exclusively AC systems, the practical value of these publication is limited. Effects of system services, for example on the profitability of power plants and corresponding dependencies between different market segments, cannot be taken into account.

In the recent literature [4], [5], there are several articles that use multi-agent-based simulation as decision models for centralized control of distribution networks. However, the multi-agent-based is intended as a decentralized "intelligent" decision node to determine the best choice between various local consumers based of market incentives. The existing communication problems, reliability and performance in network-related events are not considered [6].

The existing approaches have in common that the application of multi-agent-based simulation is limited almost exclusively to market analysis, see for example [7], [8], [9]. The underlying physical level of energy networks and the involved communication technologies and resulting issues of network control are not considered appropriately.

\section{CONCLUSION}

In summary, with the illustrated approach of multi-agent based simulation the following objectives are addressed:

- Demonstrating the feasibility of decentralized grid control by independently operating units

- Providing a fundamental basis for the physical simulation of analog power grids in a digitally operating agent system

- Design a standardized system architecture for holonic agent-based simulation systems in three levels (Physics multimodal infrastructures, communications, economic actors)

The described simulation approach integrates the required communication systems and various economic stakeholder groups in addition to the physical network level. It should moreover be possible to compare potential migration paths, regulatory incentives and economic impact in alternative scenarios.

\section{REFERENCES}

[1] A. Schwaiger, Modellierung, Simulation und Vergleich individuellen Konsumentenverhaltens mit probabilistischen Holonen. Dissertation. Universität des Saarlandes, 2006.

[2] F. Sensfuß, M. Ragwitz, M. Genoese, and D. Möst, Agent-based simulation of electricity markets: A literature review. Working paper sustainability and innovation, No. S5/2007, 2007.

[3] A. Weidlich, D. Veit, „A critical survey of agent-based wholesale electricity market models“, Energy Economics 30 (2008), pp. 17281759, 2008.

[4] D. Young, S. Poletti, O. Browne, Can Agent-Based Models Forecast Spot Prices in Electricity Markets? Evidence from the New Zea-land Electricity Market, January 24th, 2012.

[5] A. Pahwa, S.A. DeLoach, S. Das, B. Natarajan, X. Ou, D. Andresen, N. Schulz, S. Singh, Holonic Multi-agent Control of Power Distribution Systems of the Future, CIGRE US National Committee, Grid of the Future Symposium, 2012.

[6] P. Vytelingum, T. D. Voice, S. D. Ramchurn, A. Rogers, and N. R. Jennings, Agent-based Micro-Storage Management for the Smart Grid, Proc. of 9th Int. Conf. on Autonomous Agents and Multiagent Systems (AAMAS 2010), May, 10-14, 2010, Toronto, Canada, 2010. 
[7] M. S. Rahman, M. A. Mahmud, H. R. Pota, M. J. Hossain, Distributed multi-agent scheme for reactive power management with renewable energy“, Energy Conversion and Management, 88 (2014), pp. 573-581, 2014.

[8] T. Wittmann, T. Bruckner: Agentenbasierte Modellierung urbaner Energiesysteme. In: Wirtschaftsinformatik 49 (5), pp. 352-360, 2007.

[9] D. Möst, W. Fichtner, M. Ragwitz, D. Veit, New methods for energy market modelling. Proceedings of the First European Workshop on Energy Market Modelling using Agent-Based Computational Economics. 2008.

[10] S. Lamparter, S. Becher, J.-G. Fischer, An agent-based market platform for Smart Grids. In: Proceedings of the 9th International Conference on Autonomous Agents and Multiagent Systems (AAMAS '10), pp. 16891696, 2010. 